\title{
Enhanced osteoblastic adhesion through improved wettability on polarized hydroxyapatite
}

\author{
Miho NAKAMURA, ${ }^{\dagger}$ Akiko NAGAI, Toshinori OKURA, ${ }^{*}$ Yasutaka SEKIJIMA, \\ Teuvo HENTUNEN** and Kimihiro YAMASHITA \\ Institute of Biomaterials and Bioengineering, Tokyo Medical and Dental University, \\ 2-3-10 Kanda-Surugadai, Chiyoda, Tokyo 101-0062 \\ ${ }^{*}$ Environmental and Energy Chemistry, Kogakuin University, 2665-1 Nakano-cho, Hachioji-shi, Tokyo 192-0015 \\ ** Institute of Biomedicine, University of Turku, Kiinamyllynkatu 10, 20520 Turku, Finland
}

\begin{abstract}
Osteoblasts are susceptible to the surface characteristics of bioceramics and stimulation from outside the cells. The purpose of this study was to evaluate the effects of electrical polarization on surface characteristics and osteoblastic adhesion. The surface characteristics revealed that electrical polarization had no effect on surface roughness, crystallinity, and constituent elements. According to contact angle measurement, electrically polarized HA, which provides two kinds of surfaces, negatively charged $\mathrm{HA}$ (N-HA) and positively charged HA (P-HA), was even more hydrophilic than that of normal HA (O-HA). Morphological observations and quantitative analyses revealed that the typical adhered cells had a round shape on the O-HA but had a spindle spreading configuration on the N-HA and the P-HA $1 \mathrm{~h}$ after seeding. After $3 \mathrm{~h}$ of cultivation, the rate of the number of spread cells on the O-HA increased and approached that of the N-HA and P-HA. However, the cell areas positively stained for actin, which indicates the degree of cell spreading, were distinctly larger on the N-HA and P-HA than that on the O-HA. The number of focal adhesions per cell was also less than that on the N-HA and P-HA. To conclude, the polarization improved the wettability of the HA surface, which consequently affected osteoblastic adhesion, especially spreading.
\end{abstract}

(อ2010 The Ceramic Society of Japan. All rights reserved.

Key-words : Hydroxyapatite, Polarization, Wettability, Osteoblast, Adhesion

[Received February 15, 2010; Accepted March 23, 2010]

\section{Introduction}

Adhesion of the osteoblasts to inorganic biomaterials generally plays a predominant role in the regulation of the subsequent differentiation and formation of the extracellular matrix following spreading. ${ }^{1)}$ Osteoblast adhesion is primarily the result of two subjects, namely the surface characteristics of the biomaterials involved and stimulation from outside the cells. The former subject, surface characteristics, including the topography, constituent elements, functional group, and wettability of biomaterials affect osteoblast attachment and adhesion. ${ }^{2), 3)}$ The surface characteristics were reportedly affected by surface roughness, surface crystallinity, ${ }^{2)}$ the constituent elements at the surface, and the incorporation of ions such as carbonate or fluorine. ${ }^{4), 5)}$ In addition, electron-induced surface energy modifications such as photoluminescence and surface photovoltage spectroscopy were also effective in the improvement of the surface characteristics. ${ }^{6}$ ) The latter subject, stimulation from outside the cells, which includes electrical stimulation such as capacitive coupling, inductive coupling, and combined electromagnetic fields, affect osteoblast attachment, adhesion, and motility. ${ }^{6}$

Cell shapes on substrates are dependent on the integrinmediated cytoskeletal and signal transduction molecules, such as actin filaments and vinculin, ${ }^{7,8)}$ and are important during cell-substrate adhesion for subsequent cell behaviors such as proliferation and differentiation. ${ }^{5), 9)}$ Vinculin molecules are involved in the cytoplasmic domain of focal adhesion and seem

\footnotetext{
Corresponding author: M. Nakamura; E-mail: miho.bcr@tmd. ac.jp
}

to be responsible for transmitting signals from integrins on the cell surface to cytoskeletal actin filaments by the formation of focal adhesions. Because cell adhesion that is mediated via focal adhesions undergoes dynamic changes in structure and molecular properties from dot-like focal complexes to matured focal contacts, the number, the size, and the localization in the adhered cells are important. ${ }^{9)}$ Therefore, we concentrated on the adhered cell shapes that were indicated by the actin structure and vinculin localization in order to study osteoblast adhesion.

We have demonstrated that the charged surfaces on hydroxyapatite $(\mathrm{HA})^{10)-13)}$ induced by polarization enhanced osteoconductive capabilities ${ }^{14)-17)}$ and protein adsorption. ${ }^{18), 19)}$ Additionally the polarized HA accelerated the blood vessel regeneration of a vascularly injured model ${ }^{20)}$ and epidermal recovery from full-thickness skin wounds in vivo. ${ }^{21)}$ Based on the aforementioned results of the enhancement of tissue regeneration both of hard tissue and soft tissue in vivo, the polarization treatment is considered to have effects on cell behaviors. The initial adhesion and motility of osteoblast were accelerated in vitro. ${ }^{22), 23)}$ Considering two important subjects in cell adhesion, including the surface characteristics of the materials and stimulation from outside the cells, we recently focused on the surface characteristics of polarized HA and the effects of electrical stimulation by polarization on primary osteoblast behavior in vitro.

\section{Experimental procedure}

\subsection{Surface characterization}

HA powder synthesized from the analytical-grade reagents of calcium hydroxide and phosphoric acid by the wet method. The HA powder was calcined at $850^{\circ} \mathrm{C}$ and pressed in a mold at 
$200 \mathrm{MPa}$. The HA compacts were sintered in a saturated water vapor atmosphere at $1250^{\circ} \mathrm{C}$ for $2 \mathrm{~h}$. The sintered HA specimens were electrically polarized with a pair of Pt electrodes at $400^{\circ} \mathrm{C}$ in DC electric fields of $5 \mathrm{kV} \cdot \mathrm{cm}^{-1}$ for $1 \mathrm{~h}$. The surface roughness of HA specimens were analyzed using a laser microscope. A total of 15 measurements on each specimen were performed to obtain an average.

Specimens with an average density of $98 \%$ and an Ra value of 0.05 were used for surface characterization. X-ray diffraction (XRD) measurements of normal HA (O-HA), the negatively charged HA (N-HA) and the positively charged HA (P-HA) surfaces were performed for phase analysis. X-ray photoelectron spectroscopy (XPS) measurements were performed for compositional analysis. Contact angle measurements were performed on the HA specimens using distilled and deionized water. Contact angles were calculated by Young's equation: $\gamma_{\mathrm{SV}}=\gamma_{\mathrm{LV}} \cos \theta+\gamma_{\mathrm{SL}}$, where subscripts $\mathrm{S}$, L, and $\mathrm{V}$ refer to solid, liquid, and vapor.

\subsection{Cell adhesion assay}

The bone marrow cells isolated from the tibiae and femurs of $\mathrm{C} 57 \mathrm{Bl} / 6 \mathrm{~J}$ mice (female, 8-12-week-old) ${ }^{24)}$ were maintained for $7 \mathrm{~d}$ in osteoinductive culture medium, $\alpha$-MEM including $15 \%$ FCS, $100 \mathrm{IU} / \mathrm{ml}$ penicillin, $100 \mu \mathrm{g} / \mathrm{ml}$ streptomycin, $10 \mathrm{nM}$ dexamethasone, $50 \mu \mathrm{g} / \mathrm{ml}$ ascorbic acid, and $10 \mathrm{mM}$ sodium $\beta$ glycerophosphate in a humidified atmosphere of $95 \%$ air and $5 \%$ $\mathrm{CO}_{2}$ at $37^{\circ} \mathrm{C}$. The cells were seeded at a density of $1 \times 10^{4}$ cells onto the HA, which were sterilized with $70 \%$ ethanol, and immersed in the culture medium for $30 \mathrm{~min}$. At $1 \mathrm{~h}$ and $3 \mathrm{~h}$ after seeding, the cells on the HA surfaces were fixed with $4 \%$ paraformaldehyde and immunohistochemically stained. From the fluorescence actin staining, the cell morphology was categorized into three types: round, semi-spread, and well spread. ${ }^{25)}$ The first category, round, shows the cells just attached with a circular or spherical shape. The second category, semi-spread, shows the cells slightly spread with no stress fiber or lamellipodia structure. The third category, well spread, shows the cells with stress fiber or lamellipodia structure. In addition, the cell areas positively stained for actin and the number of focal adhesions positively stained for vinculin were measured using MetaMorph ${ }^{\circledR}$ software.

\section{Results}

\subsection{Characterization}

As shown in Fig. 1, the XRD patterns of the O-HA, N-HA, and P-HA were highly consistent with the published data of HA (JCPDS No. 9-432), indicating that the HA surfaces consisted of a single phase of hexagonal HA before and after polarization. XPS survey-scan spectra of the O-HA, N-HA, and P-HA surfaces are shown in Fig. 2. By comparison with the published data of XPS spectra, ${ }^{26)}$ no differences in the representative XPS spectra were found among the O-HA, N-HA, and P-HA, indicating that no significant changes occurred in the elements on the XPS analytical level during polarization. Only peaks corresponding to some $1 \mathrm{~s}$ and $2 \mathrm{p}$ orbital electrons of oxygen, calcium, phosphorus, and carbon were clearly observed on the XPS spectra of the HA surfaces.

Figure 3 shows the contact angle values obtained for the three different type of HA surfaces. The O-HA presented the highest values, indicating that the O-HA surface was a more hydrophobic material, while the N-HA and the P-HA presented lower angles, thus being more hydrophilic. The O-HA showed a contact angle of $60^{\circ}$, while the N-HA and P-HA displayed a contact angle of

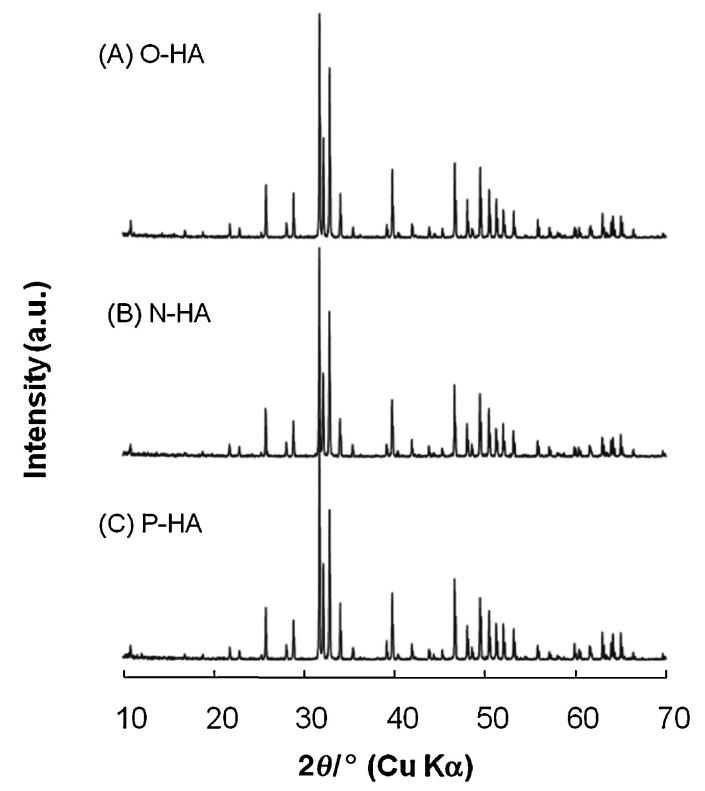

Fig. 1. XRD patterns of O-HA (A), N-HA (B), and P-HA (C). The patterns were matched to the published data of HA and demonstrated that the surfaces of the HA consisted of a single phase of hexagonal HA.

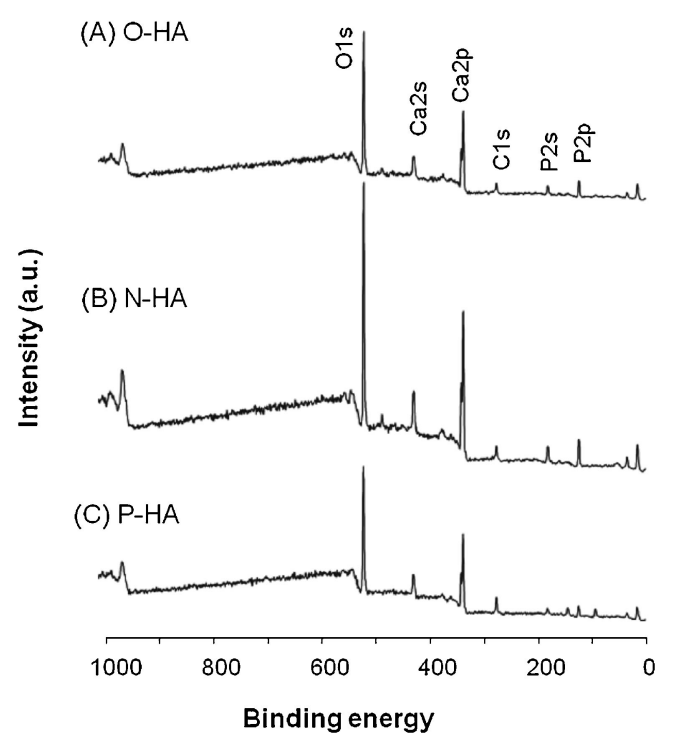

Fig. 2. Spectra of XPS survey scan of O-HA (A), N-HA (B), and P-HA (C). The representative spectra show the peaks corresponding to the same $\mathrm{s}$ and $\mathrm{p}$ orbitals of oxygen, calcium, phosphorus, and carbon.

$35^{\circ}$. These results indicated that the wettability improved on both the N-HA and P-HA.

\subsection{Cell evaluation}

Actin and vinculin labeling of the cells after seeding onto the HA for $1 \mathrm{~h}$ and $3 \mathrm{~h}$ were shown in Fig. 4. Cytoskeletal organization and formation of focal adhesions on the O-HA, N-HA, and P-HA were analyzed by immunofluorescence using antibodies directed against actin and vinculin.

During cultivation $1 \mathrm{~h}$ after cell seeding, the cells that adhered on the O-HA showed a round or spherical configuration. The localization of vinculin was equally observed in an accumulation of actin filaments in the spherical-shaped cells on the O-HA. The cells that adhered on the N-HA and P-HA already showed a 


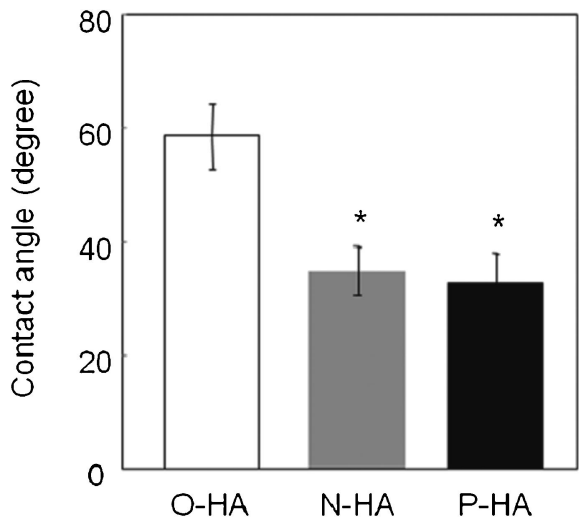

Fig. 3. Contact angle values of O-HA, N-HA, and P-HA using distilled and deionized water. The O-HA presented the highest values, indicating that the normal HA surface was a more hydrophobic material, while the $\mathrm{N}-\mathrm{HA}$ and P-HA presented a lower angle, being a more hydrophilic material.

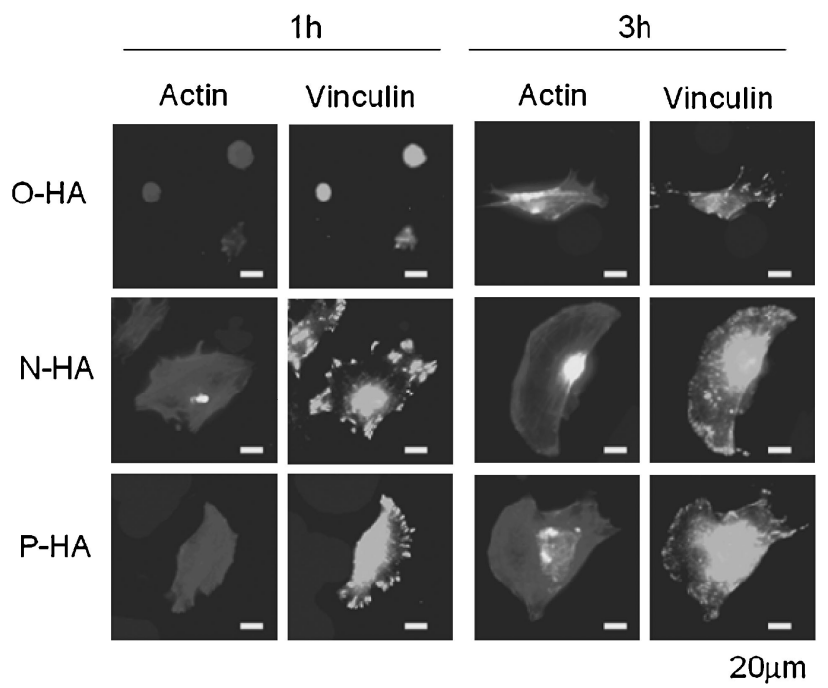

Fig. 4. Morphology of the adhered osteoblastic cells on O-HA, N-HA, and P-HA. Actin and vinculin fluorescence images of the cells cultured on the HA surfaces for $1 \mathrm{~h}$ and $3 \mathrm{~h}$ are shown.

spindle shape after $1 \mathrm{~h}$ of cultivation. As observed from the images of actin staining, the stress fiber formation was significantly stronger on the N-HA and P-HA than that on the O-HA. Immunoreaction for vinculin was found in the peripheral regions of the cells that adhered on the N-HA and P-HA. Cells left for $1 \mathrm{~h}$ before fixing and staining on the N-HA and P-HA had a well spread shape and numerous focal adhesions scattered throughout their ventral surfaces. The distribution of vinculinimmunoreactive focal adhesions on the N-HA was similar to that of the P-HA. Therefore, after $1 \mathrm{~h}$ of cultivation on the N-HA and P-HA, the differences of the polarization conditions had no obvious effects on the formation of the actin stress fiber or the focal contact distribution in the cells.

During subsequent incubation after $3 \mathrm{~h}$ of cell seeding, the cells that adhered on the O-HA were spread and showed a slightly spindle-like or rectangular shape. An accumulation of actin filaments in the periphery of lozenge-shaped cells was equally observed in the N-HA and P-HA. In addition, bundles of actin fibers forming stress fibers appeared in the cells on the NHA and P-HA as the attached cells spread. Well-defined stress fibers showing a regular arrangement with particular polarities were found in some cells grown on the N-HA and P-HA. In some cells on the N-HA and P-HA, the actin filaments were mostly distributed near the edge of pseudopodia-like structures and formed weak bundles of stress fibers. Immunoreaction for vinculin was found in the peripheral regions of the cells that adhered on the O-HA, N-HA and P-HA. The distribution of vinculin-immunoreactive focal adhesions on the N-HA was similar to that of the P-HA.

Based on fluorescence actin staining, the cell morphology was categorized into three types: round, semi-spread, and well spread. There was a remarkable difference in the degree of spreading between the O-HA and the polarized HA $1 \mathrm{~h}$ after seeding (Fig. 5A). The rate of the cells categorized as round that were cultured on the O-HA was $83 \%$, while that on the N-HA and PHA was $10-22 \%$. The cells categorized as semi-spread had almost equal subpopulations on every type of HA surface; the rate was $14-43 \%$. The rate of the cells categorized as well spread cultured on the O-HA was $3 \%$, while that on the N-HA and P-HA was $41-63 \%$. These results showed that the spreading of the cells was accelerated on both the N-HA and P-HA, compared with that on the O-HA. The degree of spreading had no significant difference between the three HA specimens $3 \mathrm{~h}$ after seeding (Fig. 5B). Although a few cells categorized as round (1-5\%) or categorized as semi-spread (6-18\%) still remained, most of the cells $(77-88 \%)$ had well spread morphologies on the three HA specimen types.

To quantify the difference in the degree of cell spreading, the cell areas positively stained for actin that adhered were measured (Fig. 5C). The cell areas increased from 1 to $3 \mathrm{~h}$, which means that the cells spread and elongated on the HA substratum. The cell areas were significantly larger on the N-HA and P-HA, compared to the O-HA $1 \mathrm{~h}$ and $3 \mathrm{~h}$ after seeding. The area of the cells cultured on the polarized HA was approximately five times or two times larger than the area on the O-HA $1 \mathrm{~h}$ or $3 \mathrm{~h}$ after seeding, respectively. However, no significant differences in cell area were observed among the N-HA and P-HA.

To quantify the difference in the formation of vinculin-positive focal adhesions, the number per cell of focal adhesions were measured (Fig. 5D). The number of focal adhesions per cell increased from 1 to $3 \mathrm{~h}$ after seeding, which means that the new focal adhesions were formed on HA. The number of the vinculin-positive focal adhesions was significantly larger on the N-HA and P-HA, compared to the O-HA $1 \mathrm{~h}$ and $3 \mathrm{~h}$ after seeding.

\section{Discussion}

The measurement of contact angle revealed that the wettability of the polarized HA was higher than that of normal O-HA, showing that both the N-HA and P-HA were more hydrophilic than that of the O-HA. Wettability has been reported to depend on surface roughness or crystallinity on a solid surface. ${ }^{2)}$ Considering these factors, the present study employed HA specimens with almost equal surface roughness, crystallinity, and constituent elements at the surface. Thus, the improved wettability of the HA surfaces resulted from other factors. The modification of surface wettability on HA was reportedly achieved by A-type carbonate apatite, corresponding to the location of carbonate ions on monovalent anionic $\mathrm{OH}^{-}$sites $\left.{ }^{4}\right)$ and by fluoridated HA, incorporating fluorine in HA. ${ }^{5)}$ However, the improvement of HA surface wettability by polarization did not affect structural transformation. In contrast, electron-induced surface energy modification such as photoluminescence and 
(A)

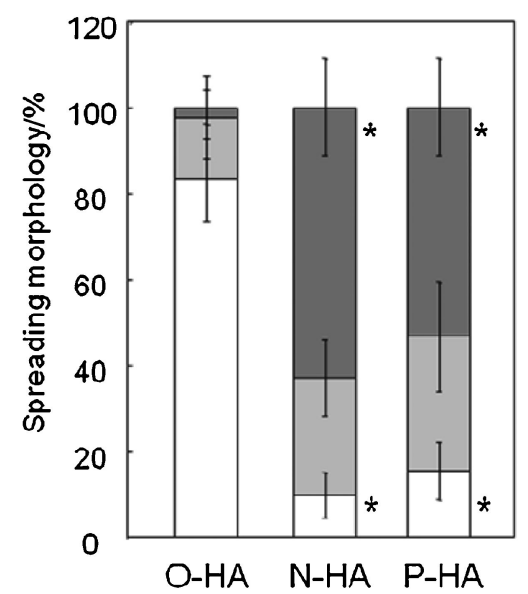

(C)

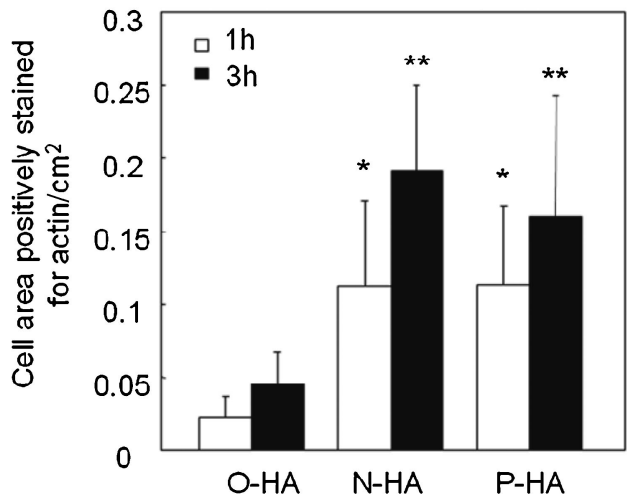

(B)

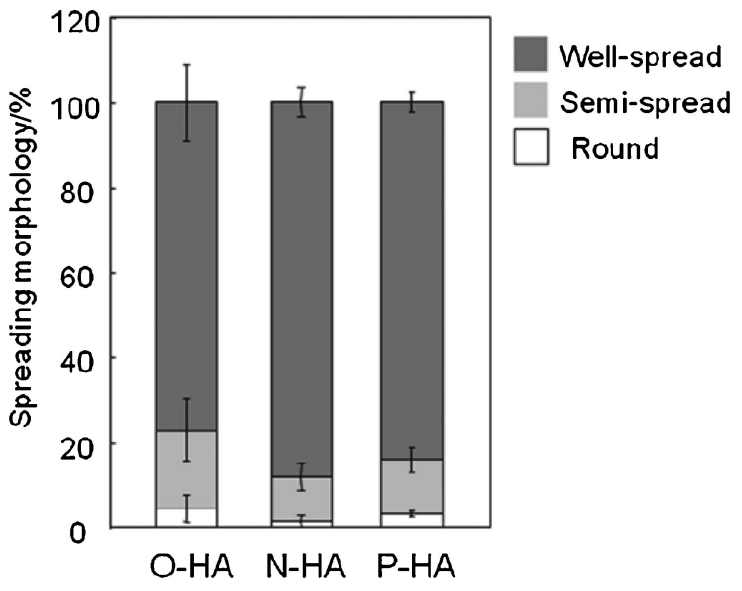

(D)

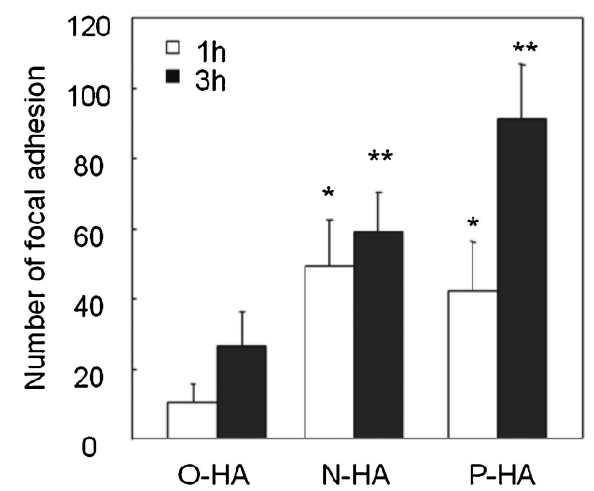

Fig. 5. The cell morphologies after seeding were categorized into three types based on fluorescence actin staining. These were round, semi-spread, and well spread. (A) Categorization of the cells on the HA specimens $1 \mathrm{~h}$ after seeding. $(*<0.005$, compared with the O-HA) (B) Categorization of the cells on the HA specimens $3 \mathrm{~h}$ after seeding. (C) To quantify the degree of cell spreading, the cell areas positively stained for actin were measured $1 \mathrm{~h}\left({ }^{*}<0.002\right.$ compared with O-HA) and $3 \mathrm{~h}\left({ }^{* *}<0.05\right.$ compared with O-HA) after seeding. (D) To quantify the formation of vinculin-positive focal adhesions, the number per cell of focal adhesions were measured $1 \mathrm{~h}\left(^{*}<0.002\right.$ compared with $\left.\mathrm{O}-\mathrm{HA}\right)$ and $3 \mathrm{~h}\left({ }^{* *}<0.05\right.$ compared with O-HA) after seeding.

surface photovoltage spectroscopy was reported as an effective method of surface energy modification of HA nanoparticle coating. ${ }^{6)}$ The possible explanation for the improved surface wettability by polarization is the surface energy modification because of polar interaction energy with water.

Morphological observation and quantitative analysis revealed that the typical adhered cells had a spherical shape on the O-HA but had a spindle spreading configuration on the N-HA and P-HA $1 \mathrm{~h}$ after seeding. After $3 \mathrm{~h}$ cultivation, the rate of the number of cells spreading on the O-HA increased and approached that on the N-HA and P-HA. However, the cell areas positively stained for actin, which showed the degree of cell spreading on the HA specimens, were distinctly larger on the N-HA and P-HA than that on the O-HA $1 \mathrm{~h}$ and $3 \mathrm{~h}$ after seeding. These results demonstrated that the charges induced on the HA surfaces affected the percentages of the number of initial cells spreading against the total number of adhered cells and on the degree of cell spreading on each cell.

The focal adhesions were highlighted by immunocytochemistry using monoclonal antibodies against vinculin. Two types of cells stained for vinculin appeared. The first appearance of vinculin staining was observed in round and spherical cells, which approximately colocalized at nuclei and actin filaments. The second appearance of vinculin staining was observed in spread cells, which localized both at the cytoplasm with a dot-like structure and at the periphery with a larger spot-like structure. More than half of the cells on the O-HA showed the first type of vinculin staining and had a small number and size of focal adhesion $1 \mathrm{~h}$ after seeding. On the other hand, almost all cells on the N-HA and P-HA showed the second type of vinculin staining and had a large number of focal adhesion $1 \mathrm{~h}$ after seeding. When the cells were left for $3 \mathrm{~h}$ before fixing and staining, the majority of the cells on the O-HA, N-HA, and P-HA showed the second type of vinculin staining and had matured large focal adhesion $3 \mathrm{~h}$ after seeding.

The number and localization of the vinculin-positive focal adhesions are important in adhered cells. The production and assembly of vinculin-positive focal adhesions is an indicator of biocompatibility and stability for cell behaviors such as adhesion, migration, growth and differentiation. The results of the vinculin localization on the N-HA and P-HA suggested that both of the polarized HA surfaces were suitable for the formation of focal complexes and subsequent focal adhesions. These benefits could contribute to cell behaviors on the polarized HA. 


\section{Conclusions}

In conclusion, this study provides two new items of information on polarized HA surface characteristics and cell behavior on polarized HA. First, polarization improved the wettability of the HA surface. Second, the improvement of surface wettability consequently affected osteoblastic adhesion, especially spreading.

\section{References}

1) A. Okumura, M. Goto, T. Goto, M. Yoshinari, S. Masuko, T. Katsuki and T. Tanaka, Biomaterials, 22, 2263-2271 (2001).

2) M. Rouahi, E. Champion, P. Hardouin and K. Anselme, Biomaterials, 27, 2829-2844 (2006)

3) Z. Schwartz and B. D. Boyan, J. Cell. Biochem., 56, 340-347 (1994).

4) S. A. Redey, M. Nardin, D. Bernache-Assolant, C. Rey, P. Delannoy, L. Sedel and P. J. Marie, J. Biomed. Mater. Res., 50, 353-364 (2000).

5) Y. Wang, S. Zhang, X. Zeng, L. L. Ma, K. A. Khor and M. Qian, J. Biomed. Mater. Res. A, 84, 769-776 (2008).

6) D. Aronov, A. Karlov and G. Rosenman, J. Eur. Ceram. Soc., 27, 4181-4186 (2007).

7) E. A. Clark and J. S. Brugge, Science, 268, 233-239 (1995).

8) S. Miyamoto, H. Teramoto, O. A. Coso, J. S. Gutkind, P. D. Burbelo, S. K. Akiyama and L. M. Yamada, J. Cell Biol., 131, 791-805 (1995).

9) A. Ben Ze'ev, S. R. Farmer and S. Penman, Cell, 21, 365-372 (1980).

10) K. Yamashita, N. Oikawa and T. Umegaki, Chem. Mater., 8, 2697-2700 (1996).

11) K. Yamashita and S. Nakamura, J. Ceram. Soc. Japan, 113, $1-$ 9 (2005).

12) K. Iwasaki, Y. Tanaka, M. Nakamura, A. Nagai, K. Hashimoto,
Y. Toda, K. Katayama and K. Yamashita, J. Am. Ceram. Soc., 91, 3943-3949 (2008)

13) Y. Tanaka, T. Iwasaki, M. Nakamura, A. Nagai, K. Katayama and K. Yamashita, J. Appl. Phys., 107, 014107 (2010).

14) S. Itoh, S. Nakamura, M. Nakamura, K. Shinomiya and K. Yamashita, Biomaterials, 27, 5572-5579 (2006).

15) W. Wang, S. Itoh and K. Yamashita, Acta Biomater., 5, 31323140 (2009).

16) S. Nakamura, T. Kobayashi, M. Nakamura and K. Yamashita, J. Mater. Sci. Mater. Med., 20, 99-103 (2009).

17) S. Nakamura, T. Kobayashi, M. Nakamura, S. Itoh and K. Yamashita, J. Biomed. Mater. Res. A, 92, 267-275 (2010).

18) M. Nakamura, S. Nakamura, Y. Sekijima, K. Niwa, T. Kobayashi and K. Yamashita, J. Biomed. Mater. Res. A, 79, 627-634 (2006).

19) M. Nakamura, K. Niwa, S. Nakamura, Y. Sekijima and K. Yamashita, J. Biomed. Mater. Res. B, 82, 29-36 (2007).

20) A. Nagai, K. Yamashita, M. Imamura and H. Azuma, Life Sci., 82, 1162-1168 (2008).

21) R. Okabayashi, M. Nakamura, T. Okabayashi, Y. Tanaka, A. Nagai and K. Yamashita, J. Biomed. Mater. Res. B Appl. Biomater., 90, 641-646 (2009).

22) M. Nakamura, A. Nagai, Y. Tanaka, Y. Sekijima and K. Yamashita, J. Biomed. Mater. Res. A, 92, 783-790 (2010).

23) M. Nakamura, A. Nagai, T. Hentunen, J. Salonen, Y. Sekijima T. Okura, K. Hashimoto, Y. Toda, H. Monma and K. Yamashita, ACS Appl. Mater. Interfaces, 1, 2181-2189 (2009).

24) M. P. Valta, T. Hentunen, Q. Qu, E. M. Valve, A. Harjula, J. A. Seppanen, H. K. Vaananen and P. L. Harkonen, Endocrinology, 147, 2171-2182 (2006).

25) S. Kato, S. Kidoaki and T. Matsuda, J. Biomed. Mater. Res. A, 68, 314-324 (2004).

26) H. B. Lu, C. T. Campbell, D. J. Graham and B. D. Ratner, Anal. Chem., 72, 2886-2894 (2000). 